\title{
SPECIAL TOPICS IN ECOLOGY: ECOLOGY OF GREATER YELLOWSTONE
}
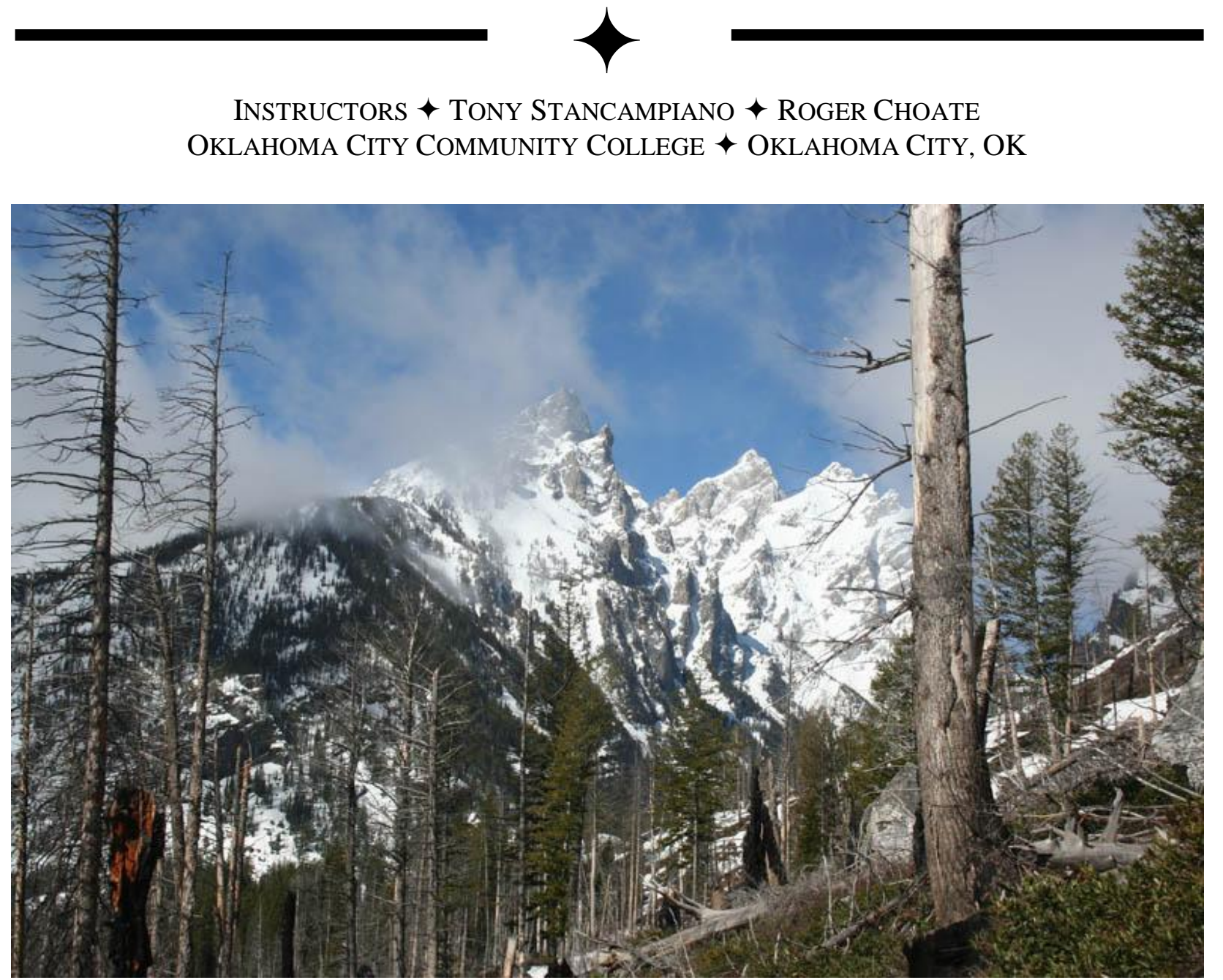

Figure 1. View of the Grand Teton Range.

\section{Class Overview}

Special Topics in Ecology: Ecology of Greater Yellowstone is a sophomore level college course emphasizing basic ecological principles with specific application to the ecosystems found in Grand Teton and Yellowstone National Parks. This course is offered during spring intersession using a hybrid format. The field portion of the course took place from May 20-29, 2011.
This year 13 students participated in the course. After completing a 4 unit online study, students embarked on a journey to the AMK Ranch with overnight camping stops in Bayfield, Colorado and Logan, Utah. The 6 day stay at AMK allowed students to experience, in the field, all of the material they had learned in the previous 2 weeks in the online portion of the class. The online learning consists of a series of lectures and associated exercises providing knowledge from multiple areas including: 


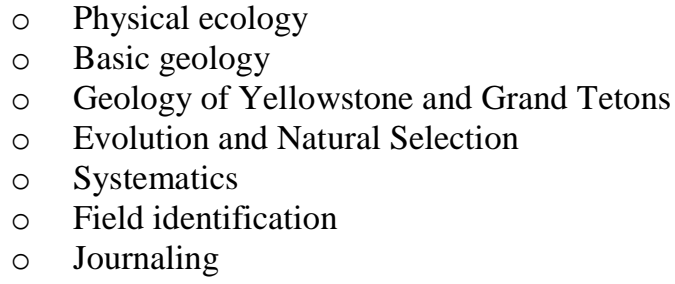

We spend each day, while in Wyoming, on a series of hikes in areas that exhibit the features, flora, and fauna discussed in the online sessions. Weather permitting, we prefer to split our time between the two parks on an every other day schedule.

In terms of logistics, the AMK is ideally located to serve as our headquarters. This setting is truly inspirational to students. The raw beauty of this locale provokes a spiritual feeling and almost palpable calling to investigate. Students learn so much more than the science. Cooperation, tolerance, scheduling, budgeting, meal planning and preparation, and consideration of the elements are but a few of the 'extra' skills learned while at the AMK.

Areas visited include, but are not limited to, the following:

○ Lewis Falls

- Kepler Cascades

○ Lonestar Geyser

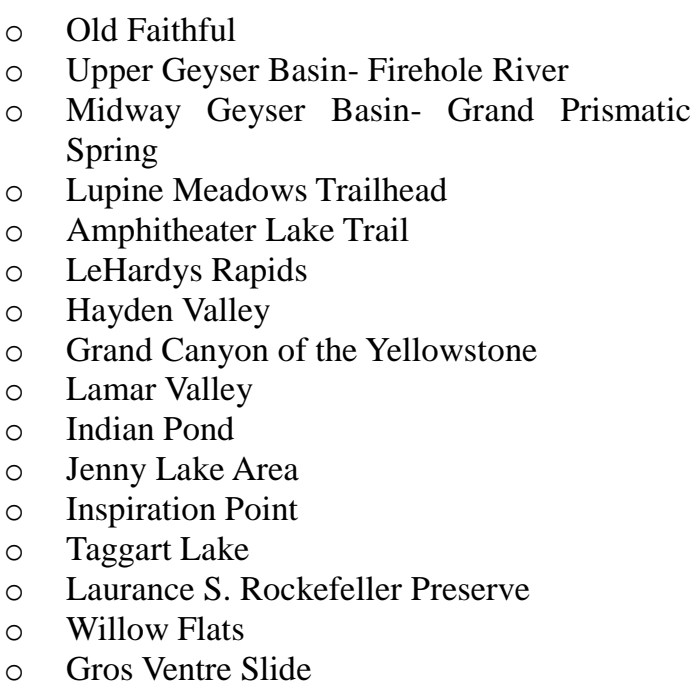

The AMK ranch and the University of Wyoming make this opportunity possible for students at OCCC. Dr. Hank Harlow and his staff, particularly Celeste Havener, facilitate our housing and accommodations at the AMK. Upon our arrival, Dr. Harlow addresses the students to inform them of general information, current events, personal safety in the parks, bear biology, history of the AMK, and related ecology/biology topics.

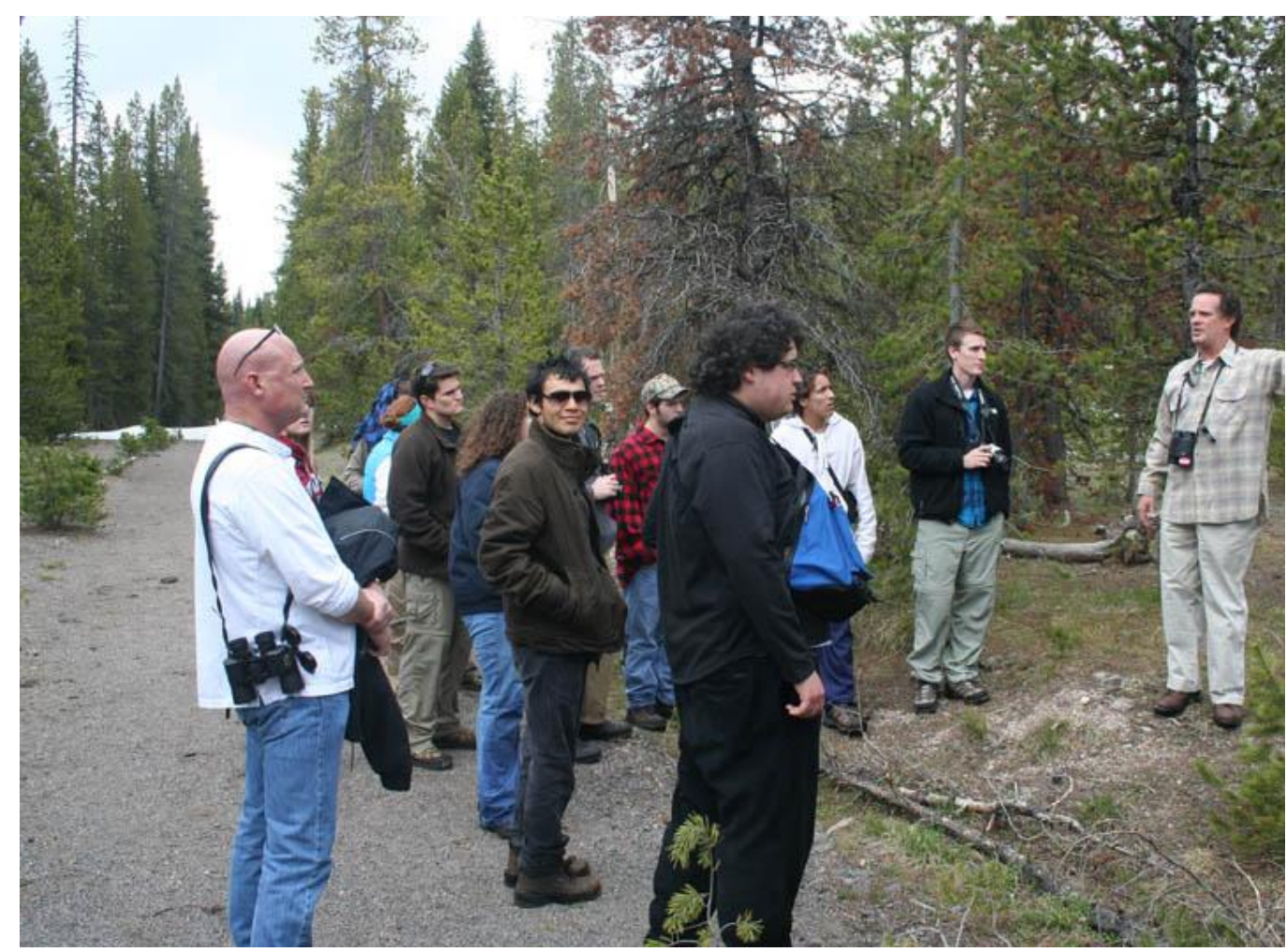

Figure 2. Tony Stancampiano giving a field lecture to students. 